\title{
Synthesis and properties of 1-aminocarbonylmethyl-5-aryl- 4-aroyl-3-hydroxy-3-pyrroline-2-ones
}

\author{
(C) Vladimir L. Gein, ${ }^{*+}$ and Evgenia V. Pastukhova \\ Perm State Academy of Pharmacy. Polevaya St., 2. Perm, 614090. Russia. \\ Phone: +7 (342) 236-90-50.E-mail: geinvl48@mail.ru
}

\begin{abstract}
${ }^{*}$ Supervising author; ${ }^{+}$Corresponding author
Keywords: 1-aminocarbonylmethyl-5-aryl-4-aroyl-3-hydroxy-3-pyrrolin-2-ones, glycinamide hydrochloride, tetrahydropyrrol-2,3-diones, three-component reactions.
\end{abstract}

\section{Abstract}

3-Hydroxy-3-pyrrolin-2-ones are fully hydrogenated heterocyclic compounds, the base of which is included in a number of biologically active substances and drugs. Scientists' interest in 3Hydroxy-3-pyrrolin-2-ones is due to the fact that, with their availability and high stability, they also have high reactivity due to the active carbonyl group in the third position of the heterocycle and easily interact with moconucleophiles. In addition, the presence of acyl and ethoxycarbonyl groups in the fourth position of the heterocycle makes it possible to obtain various condensed systems in reactions with binucleophiles, thereby making it possible to expand the range of the indicated compounds.

We set the task to prepare 5-aryl-4-acyl-3-hydroxy-3-pyrrolin-2-ones containing an aminocarbonylmethyl substituent in the first position of the heterocycle, which were not previously described in the literature. It was also supposed to establish how the obtained compounds react with hydrazine hydrate.

A series of 1-aminocarbonylmethyl-5-aryl-4-aroyl-3-hydroxy-3-pyrrolin-2-ones was obtained by setting up a three-component reaction of aroylpyruvic acid methyl ester with aromatic aldehydes and glycinamide hydrochloride in glacial acetic acid. The structure of the obtained compounds was established on the basis of IR and ${ }^{1} \mathrm{H}$ NMR spectroscopy data. Based on the data of ${ }^{1} \mathrm{H}$ NMR spectroscopy and a positive reaction with an alcoholic solution of iron(III) chloride, the predominant existence of the obtained compounds in the enol form was established.

The article describes the mechanism of three-component reactions, the structural formulas of the obtained compounds, their physicochemical properties and spectral characteristics, as well as the yields of the reaction products.

We have also studied the interaction of 1-substituted 5-aryl-4-acyl-3-hydroxy-3-pyrrolin2-ones with such a binucleophilic reagent as hydrazine hydrate. As a result of refluxing 1-aminocarbonylmethyl-3-hydroxy-4-(4-methoxybenzoyl)-5-(2-chlorophenyl)-3-pyrrolin-2-one and hydrazine hydrate in glacial acetic acid, 2-[4-(2-chlorophenyl)-3-(4-methoxyphenyl)-6-oxo-4,6-dihydropyrrolo $[3,4-\mathrm{c}]$ pyrazol-5(1H)-yl] acetamide. The article describes the proposed reaction mechanism, product yield, its physicochemical properties and spectral characteristics.

\section{References}

[1] M.D. Mashkovsky. Medicines: a guide for doctors. 16th ed., revised. and add. Moscow: New Wave. 2010. P.116, 214, 556, 616. (russian)

[2] V.L Gein. Tetrahydropyrrole- and tetrahydrofuran-2,3-diones. Perm State Pharmaceutical Academy, Perm. 2004. P.130. (russian)

[3] M.A. Maryasov, V.L. Gein. Tetrahydropyrrole-2,3-diones: monograph. Perm: Perm State Pharmaceutical Academy. 2013. P.155. (russian)

[4] L.F. Gein. Synthesis, chemical properties and biological activity of 1,4-disubstituted 5-aryl-3-hydroxy-3pyrrolin-2-ones: abstract of the dissertation of Doctor of Pharmaceutical Sciences. 2009. P.51. (russian) 